\title{
Rheological properties of magnetic and electro-active nanoparticles in non-polar liquids
}

\author{
Z. Libor, S.A. Wilson and Q. Zhang* \\ Department of Materials, Cranfield University, Bedfordshire, MK43 OAL, UK \\ *Author for correspondence q.zhang@cranfield.ac.uk
}

\begin{abstract}
The rheological properties of two non-polar liquids [silicone oil or perfluorinated oil (FC70)] containing various types of particles, barium titanate, nickel and iron-oxide, were investigated as functions of solid loading, particle size and shear rate. All the particles were synthesised in-house. The viscosities of either silicone oil or FC70 containing different solid loadings $(10,20$ and $30 \mathrm{~g} / \mathrm{l})$ were measured over the shear-rate range of $0.10-10 \mathrm{~s}^{-1}$. All the nanofluids showed shear thinning behaviour within this range and the viscosities increased with the increase of concentrations of nanoparticles and with the decrease of particle size. The highest increase of viscosity was found to be caused by nickel particles in silicone oil due to the formation of Ni network.
\end{abstract}

Keywords: magnetic nanoparticle, ferroelectric nanoparticles, rheology, silicone oil, Fluorinert, nanofluid

\section{Introduction}

Nanofluids specifically are dilute liquid suspensions of nanoparticles with at least one of their critical dimensions smaller than $100 \mathrm{~nm}$ [1] and are becoming more prominent in many areas of current technology. For example, in bio-separations, magnetic nanoparticles coated with a target-specific surface capture targets in fluids, and then are separated from the sample using a magnetic field [2]. Magnetic nanofluids offer a gentle alternative for the specific cells and molecules purification without damaging the cells and molecules. Apart from bio-separations, magnetic nanofluids can also be used in hyperthermia treatment, drug delivery and medical diagnostics [2, 3], rechargeable batteries [4], conducting paints [5], magnetic recording media, ferro-fluids and magnetic resonance imaging contrast enhancement [6]. Barium titanate $\left(\mathrm{BaTiO}_{3}\right)$ is an electroactive material, and has been widely applied to electronic industries and electro-ceramics 
industry [7, 8]. Nanofluids are under scrutiny for their heat transfer, mass transfer, wetting and spreading and antibacterial activity [1,9]. These properties of nanofluids have enormous potential for improvements in various industrial sectors, such as, chemical and process, medical, environment and energy [9].

Very small volume fractions of nanoparticles suspended in a carrier fluid have been observed to produce quite remarkable increases in macroscopic properties, such as, thermal conductivity and de electrical conductivity $[1,10]$. The most published works on nanofluids deal with the heat transfer behaviours [2, 11-15], which have been reviewed by Keblinski et al. [16] and Das et al. [12]. There have been relatively few reports on the rheological behaviour of nanofluids $[9,17-20]$ although a number of rheological studies on suspensions and/or colloids can be found in literature. In general, nanofluids could perhaps be regarded as a special category of colloids, in which low solids loading but with high enhancement and stability in rheological properties is key requirement.

The study of rheological properties of nanofluids has attracted interests of many academia and industries [21-23] because it can result in many new industrial applications. For example, in order to improve the inkjet process in direct-write technologies which allow the simplification of device manufacturing which has mainly depended upon an expensive photolithography for the patterning [21], understanding the rheological characteristics of inks such as shear thinning effects, viscoelastic property, and particle migration is necessary; the addition of a small relatively small fraction of nanoparticles to polymers, forming nanocomposites, has been noticed to induce a possible dramatic improvements of polymer properties [22-23]. Successfully making use of these materials 
depends upon a firm understanding of both their mechanical and rheological properties [22].

As a follow-up to our earlier work on nanofluids [10], in which we investigated the effect of nanoparticles dispersed in non-polar media (silicone oil and FC70 fluid) on the dc electrical conductivity of nanofluids, the aim of the present study is to achieve a more fundamental understanding of the rheological behaviour of the same nanofluids and to assess the effects of the quantity of incorporated magnetic and piezoelectric ceramic nanoparticles, particle type and size on the rheological properties of two non-polar media.

\section{Experimental}

A range of different particle types and sizes were synthesised in our laboratories for use in this study, which ensured that the particles used were in their 'pure' form and free from surface reagents, such as organic surfactants, which might sometimes be found on particles purchased from commercial suppliers. Our intension of not adding surface reagents to nanofluids to prevent the agglomeration of particles is because this allows us to observe the interactions between nanoparticles and their influence on the rheological behaviours of the base fluids.

\subsection{Nanoparticle synthesis and sample preparation}

The viscosities of six different nanofluid systems (representing combinations of three different nanoparticle types and two different carrier fluids) were measured. The nanoparticles were an electrical conductor (nickel - Ni), an electro-active ceramic (barium titanate - BT) and iron oxide (magnetite - which is one part of wüstite $\mathrm{FeO}$ and one part of haematite $\mathrm{Fe}_{2} \mathrm{O}_{3}-\mathrm{FO}$ ). BT was synthesized by hydrothermal synthesis, $\mathrm{Ni}$ 
and FO by wet chemical synthesis [24]. In all cases a narrow particle size distribution was achieved.

\subsection{Structural characterization of nanoparticles}

A range of techniques were used to characterise the nanoparticles. Transmission electron microscopy (TEM) was performed on a Philips CM 20 operating at $200 \mathrm{kV}$. Scanning electron microscopy (S-FEG SEM) was performed on a Philips XL30. X-ray diffraction (XRD) patterns were obtained using a Siemens D5005 diffractometer with CuKa radiation and a Goebel mirror. The crystallite size was determined from the X-ray line broadening using the Scherrer formula given by $D=(0.9 \lambda) /(\beta \cos \theta)$, where $\mathrm{D}$ is the average crystallite size, $\lambda$ is the X-ray wavelength used (1.5406 $\AA$ ), $\beta$ is the angular line width of half maximum intensity and $\theta$ is the Bragg angle in degrees.

\subsection{Preparation of nanofluids and rheological measurement}

Nanofluids were prepared by dispersing a known amount of oil with nanoparticles at 10 , 20 and $30 \mathrm{~g} / \mathrm{l}$ concentrations (given the bulk densities of FO, Ni and BT are 5240, 8800 and $5850 \mathrm{~kg} \mathrm{~m}^{-3}$, respectively, the volume fractions (\%) of these particles in fluids vary in the range of $0.1-0.6$ ) and then placing the suspensions in an ultrasonic water bath for 30 min. The fluids used were silicone oil (Dow Corning 200/50cS, viscosity 50cSt and specific gravity 0.96 ) and $3 \mathrm{M}^{\mathrm{TM}}$ Fluorinert FC70 perfluorinated oil (viscosity $12 \mathrm{cSt}$ and specific gravity 1.94). The latter is used sometimes in the micro-electronics industry where it provides both chemical inertia and very low electrical conductivity. No surfactants were used in the suspension formulations. The suspensions were found to be stable and there was no observable sedimentation over a period of three hours. 
Rheological measurements were performed directly after the preparation of the suspensions at room temperature $\left(25^{\circ} \mathrm{C}\right)$ using a Bohlin $\mathrm{CVO}$ rheometer (Malvern Instruments, UK). The measurements were based on the controlled shearing model at shear-rates ranging from $0.10-10 \mathrm{~s}^{-1}$. Calibrations performed against standard solutions on a daily basis over the time of this work to ensure measurement accuracy.

\section{Results and discussion}

\subsection{Nanoparticle characterization}

Fig. 1a shows the TEM image of FO nanoparticles. It shows that the particles are predominantly spherical and the size of the particles varies from 10 to $15 \mathrm{~nm}$, which is in good agreement with the value estimated by X-ray diffraction measurements (Scherrer formula at $2 \theta$ of $35.5^{\circ}$ ) [25] (Fig. 2). Moreover, FO nanoparticles are aggregates due to high surface area and magnetic dipole-dipole interactions between particles.

The morphology of the nickel sample was investigated by scanning electron microscopy (Fig. 1b). SEM images of Ni particles show that the Ni particles are also spherical with the average size between 90 and $100 \mathrm{~nm}$. The synthesized nickel nanoparticles show a narrow particle size distribution $(95 \pm 5 \mathrm{~nm})$. Due to the large surface to volume ratio and strong magnetic attraction forces, the Ni nanoparticles more tend to aggregate in order to minimize the total surface energy of the system.

A systematic study of hydrothermal synthesis of BT was carried out to understand the effects of parameters such as temperature and time on the formation of BT under alkaline conditions. The SEM micrographs (Fig. 1 c-f) of BT obtained by the hydrothermal method show that the powders contain aggregated nanoparticles with 
uniform size distribution in the range of $100-500 \mathrm{~nm}$. The average particle size obtained at $180{ }^{\circ} \mathrm{C}$ for $24 \mathrm{hrs}$ is about $100-150 \mathrm{~nm}$ (Fig. $1 \mathrm{c}$ ), at $100{ }^{\circ} \mathrm{C}$ for $52 \mathrm{hrs}$ about $200 \mathrm{~nm}$ (Fig. $1 \mathrm{~d}$ ), at $85{ }^{\circ} \mathrm{C}$ for $72 \mathrm{hrs}$ about $400 \mathrm{~nm}$ (Fig.1 e) and at $65{ }^{\circ} \mathrm{C}$ for $192 \mathrm{hrs}$ about 500 $\mathrm{nm}$ (Fig.1 f). The Ba/Ti ratio has to be 1.05:1 to achieve stoichiometric BT powder. Excess barium leads to the formation of some $\mathrm{BaCO}_{3}$ (due to the reaction with atmospheric $\mathrm{CO}_{2}$ ). The formed $\mathrm{BaCO}_{3}$ was washed out using formic acid.

FO, Ni and BT (100 and $200 \mathrm{~nm})$ particles are spherical, but BT particles are more or less cubic at larger sizes (especially at 400-500 nm).

The XRD peaks of FO (Fig. 2) could be indexed by the cubic structure of FO (JCPDS no. 19-629) indicating a high phase purity of FO and the diffraction peaks are broad owing to small crystallite size.

In the case of Ni particles (Fig. 3) three characteristic peaks for nickel $\left(2 \theta=44.5^{\circ}\right.$, $51.8^{\circ}$, and $76.4^{\circ}$ ), corresponding to Miller indices (111), (200), and (222), are observed. This reveals that the resultant particles are pure face-centred cubic (fcc) nickel (JCPDS, No. 04-0850) and only nickel is detected. Although it is known that nickel is easily oxidized to oxides, some possible oxides such as $\mathrm{NiO}, \mathrm{Ni}_{2} \mathrm{O}_{3}$ are not observed in the XRD profiles. The calculated size values for pure Ni by Scherrer formula at $2 \theta$ of $44.5^{\circ}$ are general approximates to that of SEM observation (Fig. 1b).

Figure 4 shows all the XRD patterns of the BT samples. As observed in Fig. 4 the XRD patterns fit well to the peak positions of the standard cubic phase $\mathrm{BaTiO}_{3}$ (JCPDS No.31-174). As reported by Clark et al. [26] routine XRD is sensitive to small quantities of $\mathrm{BaCO}_{3}$ occurring in the hydrothermal process of BT. It is known that in the perovskite structure, the [100] planes have the lowest surface energy [26]. The high-energy faceted 
edges of the cubic particles may be subject to preferential dissolution. In addition, the strain associated with the stabilization of the cubic structure tends to reduce the surface area of the growing particles. Therefore, the intermediate morphology consists of cubic lattice particles with a spherical morphology regained during the final stage of growth, although some larger particles still retain their cubic morphology. The final particle morphology is probably a result of the competition between the growth rate and the edge effects. The structural defects of BT nanocrystals synthesized by the hydrothermal method are primarily in the form of lattice $\mathrm{OH}^{-}$ions, which are compensated by barium vacancies created on the surfaces of individual particles to maintain electro-neutrality [26].

\subsection{Sedimentation of nanoparticles in nanofluids}

In order to investigate the possible effect of the gravity in our experiments, first of all the sedimentation time was estimated based on the force balance between the buoyancy force and the Stokes drag force. Sedimentation time is the time a particle takes to settle through a depth of $1 \mathrm{~mm}$ in nanofluid. The sedimentation time can be estimated by the Stocks law:

$$
\mathrm{V}=\left(\rho_{\mathrm{P}}-\rho_{\mathrm{L}}\right)_{\mathrm{g}} \mathrm{R}^{2} / 18 \eta
$$

Where $\mathrm{V}$ is a sedimentation velocity of particles, $\mathrm{R}$ is a particles radius, $\eta$ is the viscosity of the carried medium, $\rho_{\mathrm{P}}$ is the densities of the particles and $\rho_{\mathrm{L}}$ is the density of the medium and $\mathrm{g}$ is the acceleration rate of the gravity. The measurement of settling time was carried out by using a high speed and high magnification camera. Photos have been taken time after time up to $24 \mathrm{hrs}$. and the data were compared with a special photoprogram. This allows detecting any movement of particles in the fluid more closely than just using any sort of camera. There was no observable sedimentation over a period of 3 
hrs for all the prepared nanofluids (except for Ni-silicone oil nanofluid). The absence of surfactant gives an opportunity to use the "pure" nanofluids in further characterization without additional complexity. The stability of nanofluids generally depends on the particles' surface properties, size and shape. Agglomeration of nanoparticles occurs readily in nanofluids, effectively increasing their size and their sedimentation rate.

The measured sedimentation time of nanofluids shows that no sedimentation was visible in the FO-silicone oil nanofluid up to $8 \mathrm{hrs}$; in the Ni-silicone oil nanofluid the observable sedimentation started after $2.5 \mathrm{hrs}$ and the sedimentation of the BT-silicone oil nanofluid was observable after $3 \mathrm{hrs}$. The sedimentation of different nanofluids starts at different times due to the different particle sizes and bulk densities of suspended nanoparticles as well as the different interactions between nanoparticles. Due to the lack of any additives or surfactant, the sedimentation eventually occurs due to the agglomeration of nanoparticles. All the measurements of rheological properties did not exceed $0.5 \mathrm{hr}$ and hence the sedimentation effect was ignorable.

\subsection{Rheological behaviour}

\subsubsection{Effect of solid loading on viscosity}

Fig. 5 shows the viscosity change of silicone oil containing nanoparticles against shear rate in the range of $0.10-10 \mathrm{~s}^{-1}$. All of the suspensions show shear-thinning flow behaviour over the whole examined shear rate range.

The viscosity of FO nanofluids increases with increasing particle loading, as shown in Fig. 5a. This may be explained as: because the interaction between particles increases when the particle loading increases. When two colloidal particles approach each other, the double layer on each particle overlaps and it thus generates a repulsive 
force on each other. This repulsive force is partly affected by an attractive force due to the van der Waals interaction between the approaching particles and exhibits a power-law distance dependence whose strength depends on the dielectric properties of the interacting colloidal particles and intervening medium [27, 28].

The rheological behaviour of suspensions with different relative contents of nickel (Fig. 5b) is affected both by the volume fraction of solids and by agglomeration of nickel nanoparticles. The nickel nanoparticles are spherical and tend to form aggregates that in principle induce local changes in the viscosity of the suspension. The effects of the aggregated particles on the rheological behaviour of suspensions are quite probably nonlinear and the different loadings of nanoparticles result in only small changes to the viscosity. The investigations of the aggregated nickel suspensions in this study and in earlier ones $[29,30]$ indicate that there may be a network of particles in the carrier fluid. In organic media, such as, terpineol in [29] and silicone oil in this case, this may lead to different stabilization and sedimentation mechanisms, compared to the most-studied aqueous medium [31]. The sizes of the nickel used in previous studies were twelve [31] and three times [29] larger than that of the particles used in this study. In comparison of other two nanofluids in Fig. 5 a and c, the highest increase of viscosity is observed to be in the nickel nanoparticle suspensions in silicone oil, which indicates that strong interactions between $\mathrm{Ni}$ nanoparticles exist and such interactions become greater when the nanoparticle loading increases, which thus may lead to the formation of not only $\mathrm{Ni}$ particle aggregates but also Ni network.

Suspensions of BT in silicone oil (Fig. 5c) exhibit non-linear viscoelastic behaviour of shear thinning. This is in agreement with the observations made by Khastgir 
et al. [28], who attributed the viscoelastic behaviour to the shear rate dependence of the size and structure of aggregates of BT particles. If the system contains more than $10 \mathrm{vol} \%$ of solids, it shows the signs of plasticity which was explained as the formation of networks of particles. In our case, the volume percentage of BT particles in silicone oil is less than $1 \%$, far below $10 \%$; it is unlikely to form networks of particles in BT nanofluids. When the size of the particles decreases (BT 100-120 nm, Ni 90-100 nm and FO 10-15 nm), the specific surface area of the particles increases and there are larger numbers of nanoparticles in the suspension for a given weight loading. This clearly has an influence on the macroscopic fluid properties. However, such an influence is overshadowed by the agglomeration of primary nanoparticles. In another word, the viscosity change of the fluid containing nanoparticles is due to the role played by agglomerated particles.

The two fluid media used in this investigation have different chemical make-up, different viscosity and different density. Hence the inter-particle interaction in these media should be expected to be different. Fig. 6 ( $a, b$ and $c$ ) shows the change of viscosity of suspensions containing three types of nanoparticles in FC70 against shear rates. All of the suspensions show shear-thinning flow behaviour over the whole examined shear rate range $\left(0.10-10 \mathrm{~s}^{-1}\right)$. The viscosity increases with increasing solid loading of FO, Ni and BT indicating that the inter-particle interaction has an influence on the rheological behaviour of the suspensions. Fig. 6a indicates that the rheological behaviour of the suspensions containing the particles of FO depends mainly on the strength of nanoparticles interaction. The Ni-FC70 suspensions (Fig. 6b) do not show the greatest increase of viscosity with the increase of particles concentration as observed in 
silicone oil, indicating relatively weaker interactions between $\mathrm{Ni}$ particles and/or between Ni particles and base fluid when they are in FC70; probably the formation of the network of Ni particles does not occur. The BT-FC70 suspensions with different solid additions are all viscoelastic in the examined shear rate range (Fig. 6c).

\subsubsection{Effect of particle size on viscosity}

In general, the reduction of the average size of the solid-phase while keeping its concentration constant brings particles much closer together. In this study the interparticle spacing varies typically within a notional, 3-10 particle diameter, given a uniform suspension. Fig. 7 shows the viscosity of suspensions against shear rate in the range of $0.10-10 \mathrm{~s}^{-1}$ for (a) silicone oil and for (b) FC70 fluid suspension with $20 \mathrm{~g} / \mathrm{l}$ of solid concentrations of BT particles (size $120 \mathrm{~nm}, 200 \mathrm{~nm}$ and $500 \mathrm{~nm}$ ). All of the suspensions show shear-thinning flow character at the whole range of shear rates.

The viscosity both in silicone oil and in FC70-based nanoparticles suspensions increases with decreasing size of the BT particles. Fig. 7 shows that the size of the particles plays an important role both in the silicone oil and in the FC70 fluid-based nanoparticles suspensions. The smaller particles $(120 \mathrm{~nm}$ and $200 \mathrm{~nm})$ cause bigger increase in the viscosity, which is in agreement with some previous studies [32-34]. The flow curves (Fig. 7) show increasing shear thinning behaviour with decreasing particles size.

Fig. 8 illustrates the notional behaviour of nano-suspension with increasing shear rate. Overall it can be assumed that the rheological properties of nano-suspensions are influenced by many factors, not only by the particle concentration, size and the 
interaction between the particles and the medium, but also by higher order networking and agglomeration.

One of the latest reviews [35] shows a similar observation to those shown here, namely that the viscosity increases with the volume fraction of nanoparticles and with the size reduction. One of the highest increases in viscosity was measured by Wang et al. [36] who found an $86 \%$ increment of the effective viscosity in $\mathrm{Al}_{2} \mathrm{O}_{3}$ water-based nanofluids. The classical models which were used to predicting the viscosity of nanofluids such as Einstein's [37], Krieger and Dougherty's [38] and Nielsen's [39] could not be used to fully explain our observations.

\subsubsection{Effect of nanoparticles mixture on viscosity}

FO and Ni nanoparticles were dispersed in two types of fluids in the ratio of $1: 1$ by weight. The difference of the size of the suspended particles is $1: 10$ in this case, and the mixed nanoparticles system was found to sediment more slowly than one-type particle system with larger particles. It is believed that the smaller particles influence the "network" of larger particles, in this case, Ni particles. Even weak inter-particle attraction has an effect on the sedimentation. The van der Waals attraction between the bigger particles is different as well when the system contains small sized particles. The small particles are thought to prevent the bigger particles touching each other and mixedparticle agglomerates are energetically unfavoured [40].

Sedimentation was experimentally observable in the mixed nanoparticles system after $3.5 \mathrm{hrs}$, which is $1 \mathrm{hr}$ longer than for the pure nickel nanofluid. Our experimental result shows good agreement with the study by Thies-Weesie et al. [41] that shows that 
the sedimentation velocity is reduced under gravity for mixed uncharged spherical particles.

Fig. 9 shows the viscosity of mixed-particle suspensions against shear rate in the range $0.10-10 \mathrm{~s}^{-1}$ for (a) silicone and (b) FC70 oils with 10 and $20 \mathrm{~g} / \mathrm{l}$. The mixtures chosen were of nickel and iron-oxide nanoparticles. The silicone oil-Ni suspension has shown the largest increase of viscosity in all the examined one-type nanoparticles suspensions and FO particles were used to provide a bimodal size range. This leads to slightly reduced viscosity, when compared against the expected value at the same solid concentrations, which probably is because small FO particles prevent the formation of $\mathrm{Ni}$ network. A general observation found that the shear thinning behaviour of a pure fluid appears in the shear rate range of $<1 \mathrm{~s}^{-1}$. However, a close observation would find that shear thinning range extended to the range of $1-10 \mathrm{~s}^{-1}$ in the mixtures, which implies that the inter-particle interaction between Ni and FO probably still exists.

\section{Conclusion}

Three types of nano-particles with different sizes have been synthesised using wetchemical and hydrothermal methods. Nanofluids were prepared using silicone oil and FC70 fluid as dispersing media. The rheological characterization of these nanofluids was carried out. All of the silicone oil and FC70 suspensions (containing 10, 20 and $30 \mathrm{~g} / \mathrm{l}$ nanoparticles) show shear thinning behaviour at the shear-rate range of $0.10-10 \mathrm{~s}^{-1}$. The largest change of viscosity in silicone oil was exhibited by nickel suspensions. This result is due to the fact that the interaction of metallic $\mathrm{Ni}$ in silicone oil leads to the formation of network. In a parallel study [10] it was demonstrated that $30 \mathrm{~g} / \mathrm{l}$ nickel nanoparticles suspended in the silicone oil exhibited a DC conductivity $(0.1 \mathrm{~S} / \mathrm{m})$ that was almost 
eleven orders of magnitude greater than that of the base fluid $\left(10^{-12} \mathrm{~S} / \mathrm{m}\right)$, whereas the corresponding increase in DC conductivity for $30 \mathrm{~g} / 1$ nickel nanoparticles in FC70 (8 x $\left.10^{-12} \mathrm{~S} / \mathrm{m}\right)$ compared to the base fluid $\left(10^{-12} \mathrm{~S} / \mathrm{m}\right)$ was quite modest. BT particles with the sizes of 120, 200 and $500 \mathrm{~nm}$ suspended in silicone oil and FC70 fluid showed an increase of viscosity with decreasing particle size, indicating the increase of interactions between particles when particle size decreases. In the nanofluids containing both FO and $\mathrm{Ni}$ particles, the smaller FO particles prevent Ni particles from forming network and therefore the effect of Ni particles on the viscosity is not as obvious as in the nanofluids containing only Ni particles.

Experimental results shown in this study indicate that the viscosity of the organic fluids could be controlled within a narrow range or tuned by adding nanoparticles in the fluids.

\section{Acknowledgements}

Zsuzsanna Libor would like to acknowledge the financial support of the UK Engineering and Physical Sciences Research Council (EPSRC) under Platform Grant No. EP/D506638/1 Nanoscale Multifunctional Ferroic Materials and Devices.

\section{References}

[1] Choi S (1995) Developments Applications of Non-Newtonian Flows FED-vol 231/MD-vol 66, ed Siginer D A and Wang H P (New York: ASME)

[2] Chiang CL., Sung CS., Chen CY (2006) J Magn Magn Mater 305:483

[3] Vekas L., Bica D. and Avdeev MV (2007) China Particuology 5:43

[4] Antolini E, Ferretti M, Gemme S (1996) J Mater Sci 31:2187

[5] Eisazadeh H, Spinks G, Wallace GG (1993) Mater Forum 17:241 
[6] Zhang HT, Wu G, Chen XH, Qiu XG (2006) Materials Research Bulletin 41 : 495

[7] Ikawa H, Munekata N, Shirakami T (2002) Trans Mater Res Soc Japan 27 : 707

[8] Tokita K, Sato S (2005) Key Eng Mater 301:219

[9] Chen H, Ding Y, Tan C (2007) New J Phys $9: 367$

[10] Wilson SA, Libor Z, Skordos AA, Zhang Q (2009) J Phys D: Appl Phys 42 : 062003

[11] Masuda H, Ebata A, Teramae K, Hishiunma N (1993) Netsu Bussei Japan 4 : 227

[12] Das SK, Putra N, Roetzel W (2003) Int. J. Heat Mass Transfer $46: 851$ and (2003) Int. J. Multiphase Flow $29: 1237$

[13] Wen DS, Ding YL (2004) J Thermophys Heat Transfer $18: 481$ and $47: 5181$ and (2005) J Nanoparticle Res $7: 265$

[14] Pak BC, Cho YI (1998) Exp Heat Transfer 11:151

[15] Ding YL, Alias H, Wen DS, Williams RA (2006) Int J Heat and Mass Transfer 49:240

[16] Keblinski P, Eastman JA, Cahill DG (2005) Mater Today 8:36

[17] Das SK, Choi S, Patel HE (2006) Heat Transfer Eng 27:2

[18] Kwak K, Kim C (2005) Korea-Australia Rheology J 17:35

[19] Prasher R, Song D, Wang J (2006) Appl Phys Lett 89:133108

[20] Tseng WJ, Lin KC (2003) Mater Sci Eng A 355:186

[21] Park B. J., Park B. O., Ryu B. H., Choi Y. M., Kwon K. S., Choi H. J. (2010) J Appl Phys 108: 102803

[22] Wypych G., (1999) Handbook of Fillers; Chem Tec Publishing: Toronto

[23] Giannelis E.P. (1996) Adv Mater 8: 29

[24] Libor Z, Zhang Q (2009), Mater Chem and Phys 114 : 902 
[25] Chanda SC, Manna A, Vijayan V, Nayak Pranaba K, Ashok KM, Acharya HN(2007) Mater Lett 61:505

[26] Clark IJ, Takeuchi T, Ohtori N, Sinclair DC (1999) J Mater Chem $9: 83$

[27] Barnes HA, Hutton JF, Walters K (1993) An Introduction of Rheology (Elsevier, Amsterdam) p116

[28] Khastgir D, Adachi K (2000) Polymer 41 : 6403

[29] Tseng WJ, Chen CN (2003) Mater Sci and Eng A 347: 145

[30] Tseng WJ, Chen CN (2006) J Mater Sci 41:1213

[31] Sanchez-Herencia AJ, Hernandez N, Moreno R (2006) J Am Ceram Soc 89 : 1890

[32] Yan Y, Pal R, Masliyah J (1991) Chem Eng Sci 46 : 985

[33] Nguyen CT, Desgranges F, Roy G, Galanis N, Mare T, Boucher S, Mintsa HA (2007) Int J of Heat and Fluid Flow $28: 1492$

[34] Chen S, Oye G, Sjoblom J (2005) J of Dispersion Sci and Technol 26 : 791

[35] Murshed SMS, Leong KC, Yang C (2008) Appl Thermal Eng 28 : 2109

[36] Wang X, Xu X, Choi SUS (1999) J Thermophysics and Heat Transfer 13 : 474

[37] Einstein A (1906) Ann Physik 19 : 289

[38] Krieger JM, Dougherty TJ (1959) Trans Soc Rheology 3 : 137

[39] Nielsen LE (1970) J Appl Phys 41:4626

[40] Wilson SA (1999) PhD thesis, Cranfield University, UK.

[41] Thies-Weesie DME, Philipse AP, Lekkerkerker HNW (1996) J. Colloid and Interface Sci. $177: 427$ 


\section{Figure captions:}

Figure 1 SEM and TEM images of particles. a: FO (TEM, 10-15 nm); b: Ni (90-100 nm); c: BT (batch $24 \mathrm{hrs}$ at $180^{\circ} \mathrm{C}, 100-150 \mathrm{~nm}$ ); d: BT (batch $52 \mathrm{hrs}$ at $100^{\circ} \mathrm{C}, 200 \mathrm{~nm}$ ); e: BT (batch $72 \mathrm{hrs}$ at $85^{\circ} \mathrm{C}, 400 \mathrm{~nm}$ ); f: BT (batch $192 \mathrm{hrs}$ at $65^{\circ} \mathrm{C}, 500 \mathrm{~nm}$ ).

Figure 2 XRD pattern of FO nanoparticles

Figure 3 XRD pattern of Ni nanoparticles

Figure 4 XRD patterns of BT particles of the size range $150-500 \mathrm{~nm}$

Figure 5 The viscosity change of silicone oil containing nanoparticles against shear rate in the range of $0.10-10 \mathrm{~s}^{-1}$. The nanoparticles are (a) FO (b) Ni and (c) BT. The concentration of nanoparticles is expressed in 10,20 and $30 \mathrm{~g} / 1$ and it corresponds to 
0.043, 0.086 and $0.129 \mathrm{~mol} / \mathrm{l}$ for FO; $0.170,0.340$ and $0.51 \mathrm{~mol} / \mathrm{l}$ for $\mathrm{Ni}$; and $0.043,0.086$, and $0.129 \mathrm{~mol} / \mathrm{l}$ for BT

Figure 6 Viscosity vs. shear rate relationships in FC70 fluids containing FO (a); Ni (b); and BT (c)

Figure 7 Viscosity vs. shear rate relations of the BT-silicon oil (a) and the different size BT-FC70 fluid (b) at $20 \mathrm{~g} / \mathrm{l}$ concentration

Figure 8 Schematic graph of rheological behaviour of nanoparticles in oil

Figure 9 The viscosity-shear rate relation of the suspension containing both Ni and FO in silicon oil (a) and in FC70 fluid (b) at 10 and $20 \mathrm{~g} / \mathrm{l}$ concentrations 

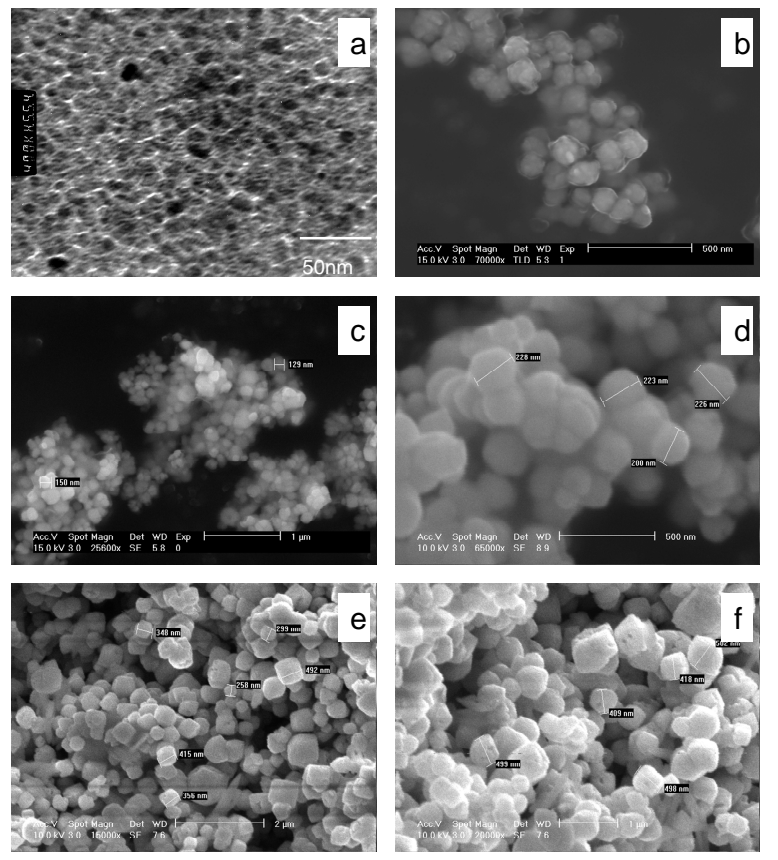

Figure 1 


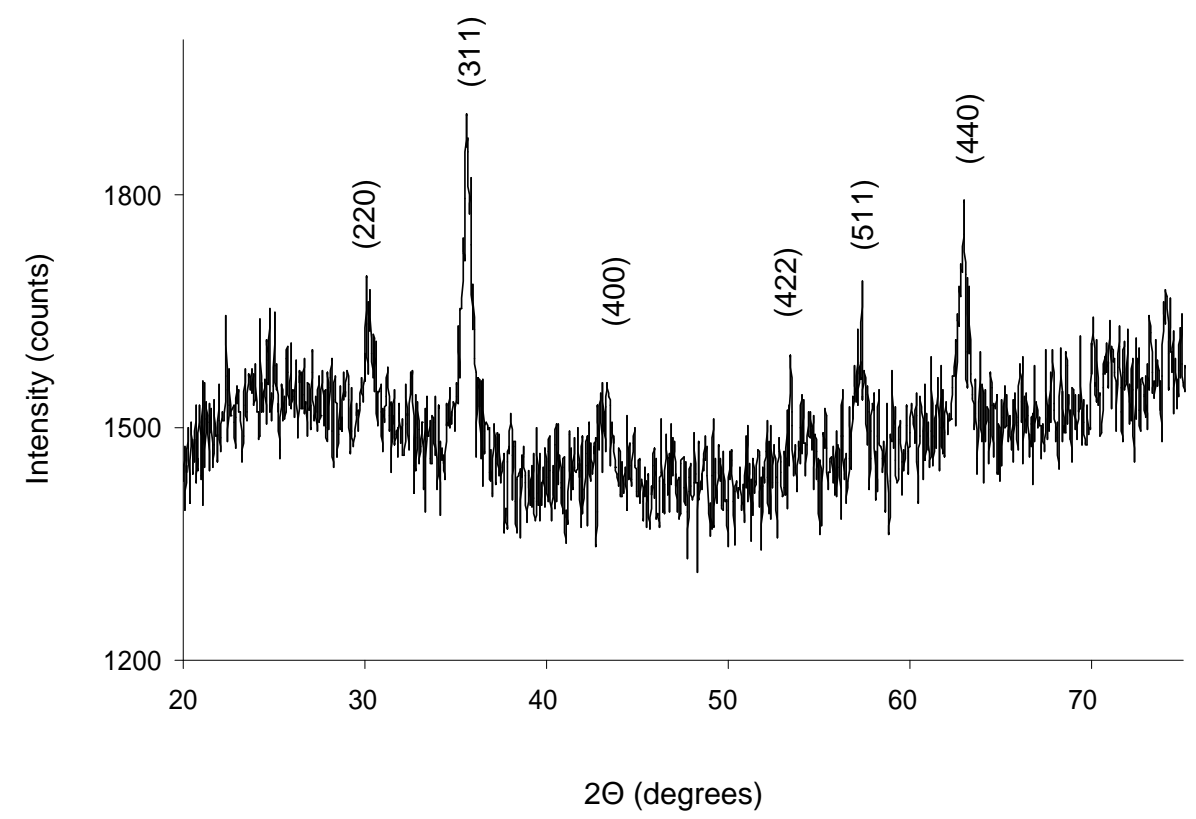

Figure 2 


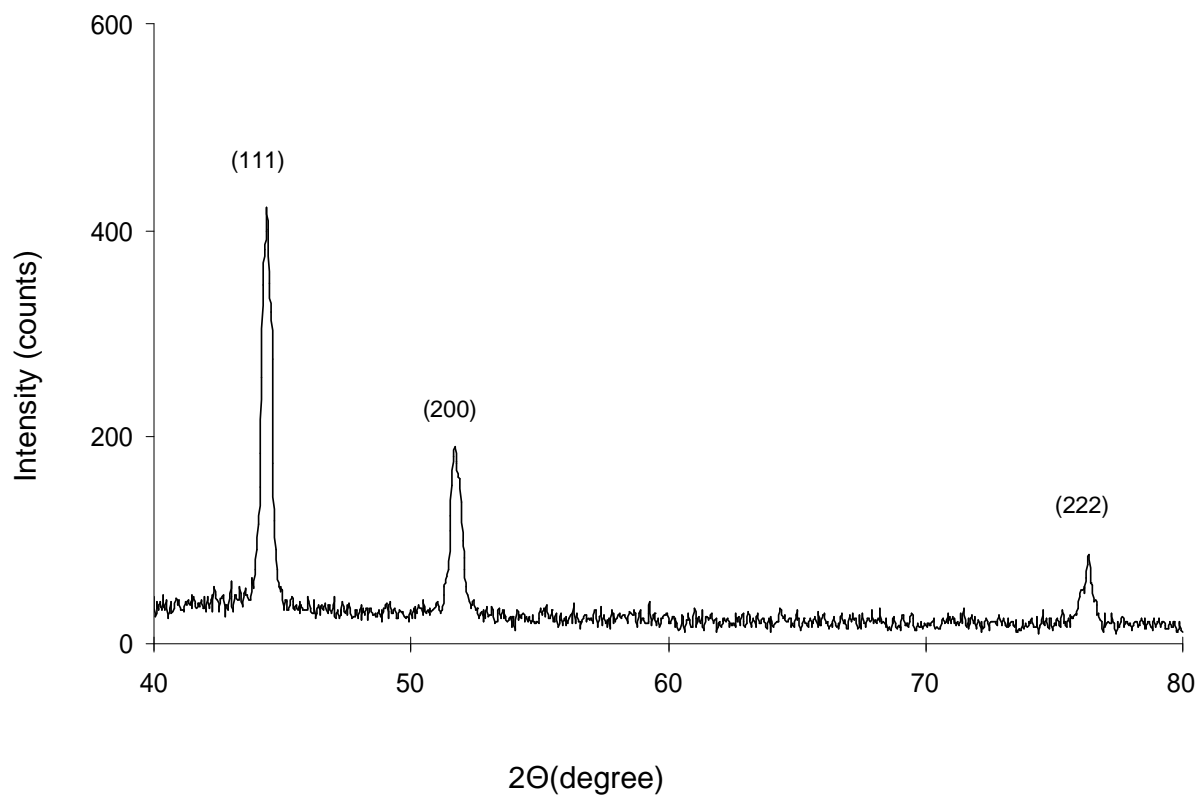

Figure 3 


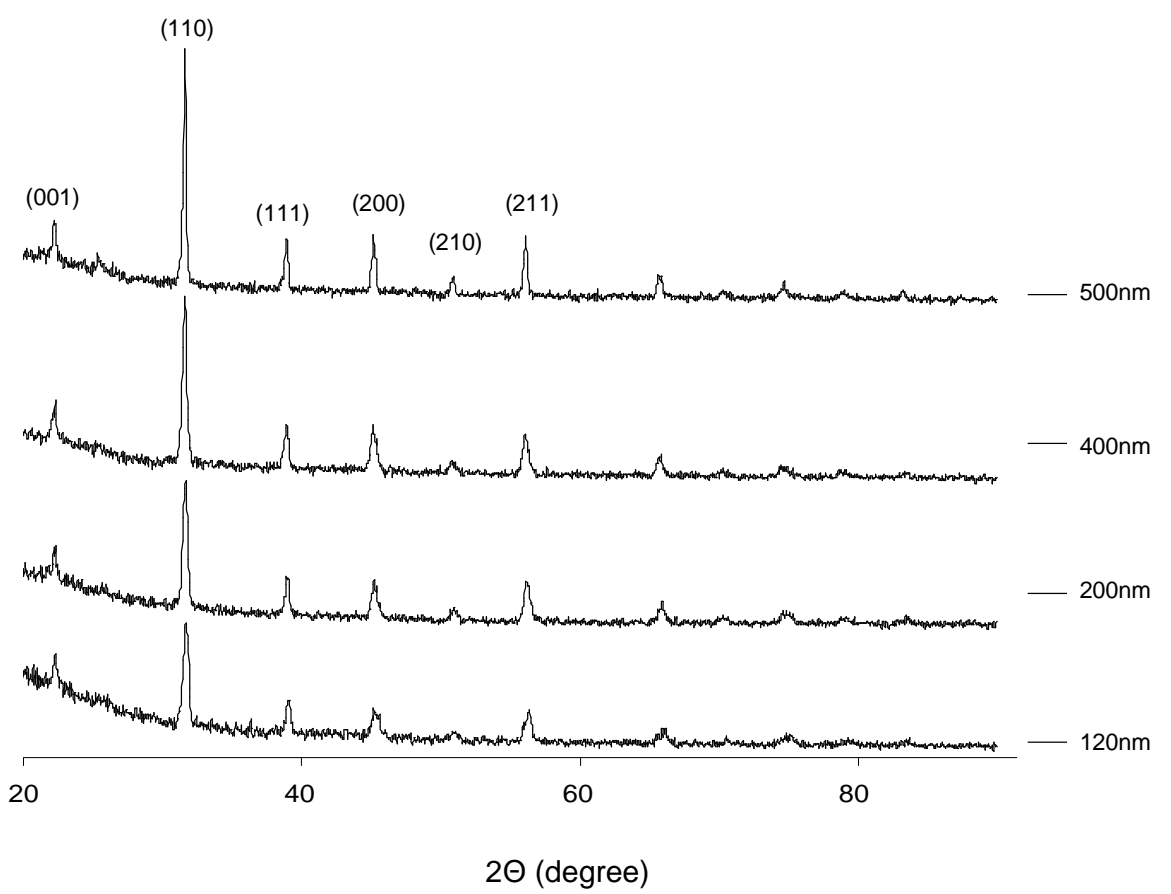

Figure 4 
(a)

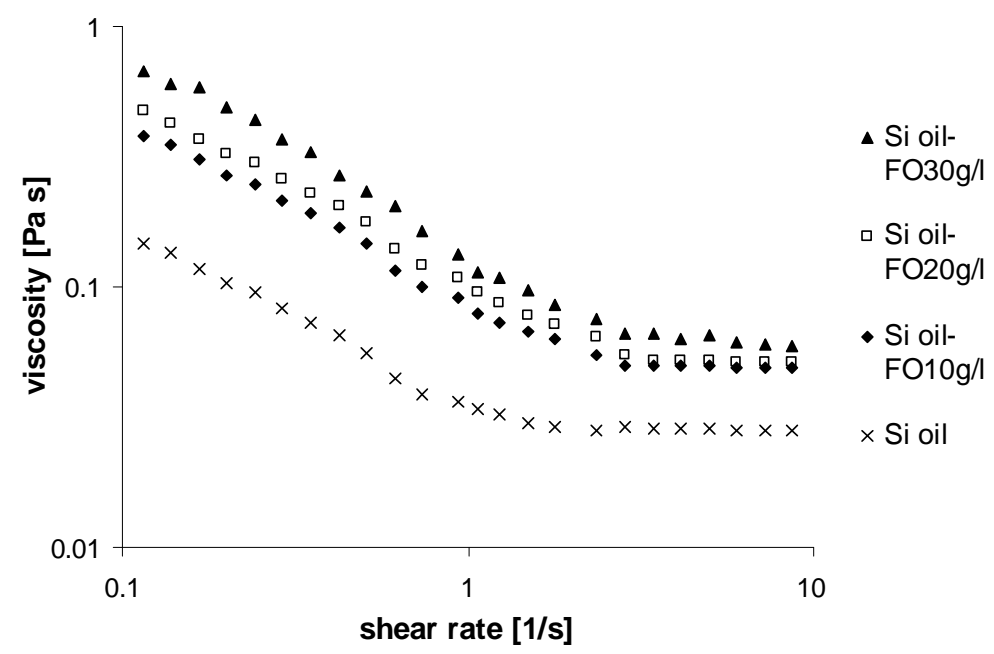

(b)

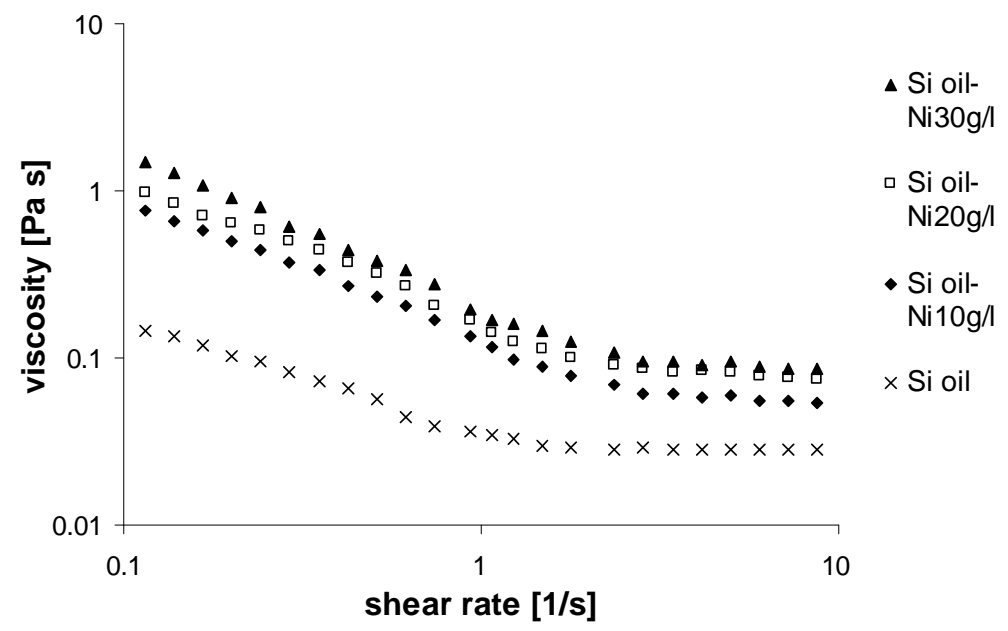

(c)

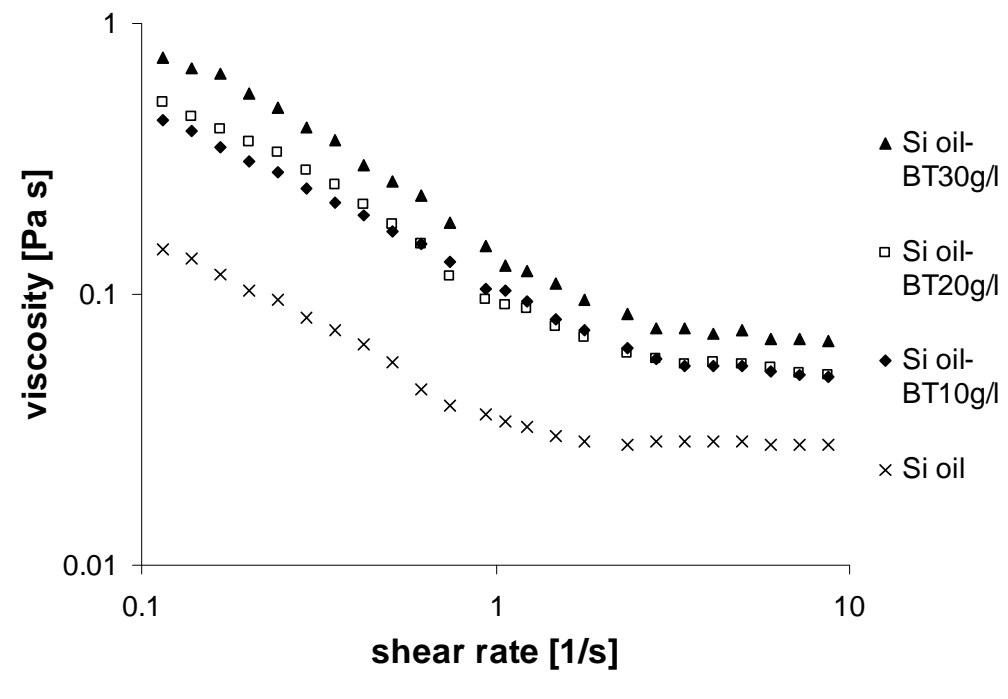

c;

Figure 5 a-c 
(a)

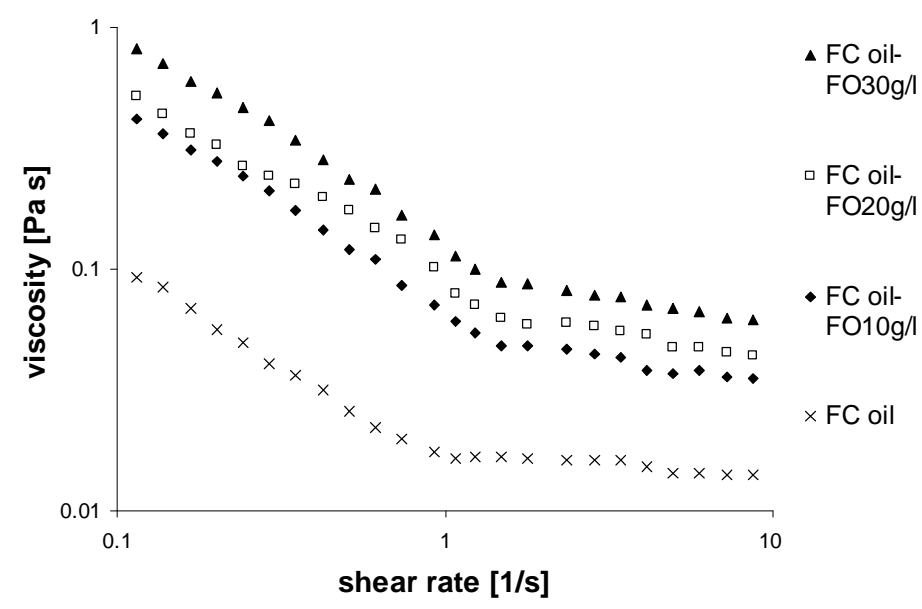

(b)

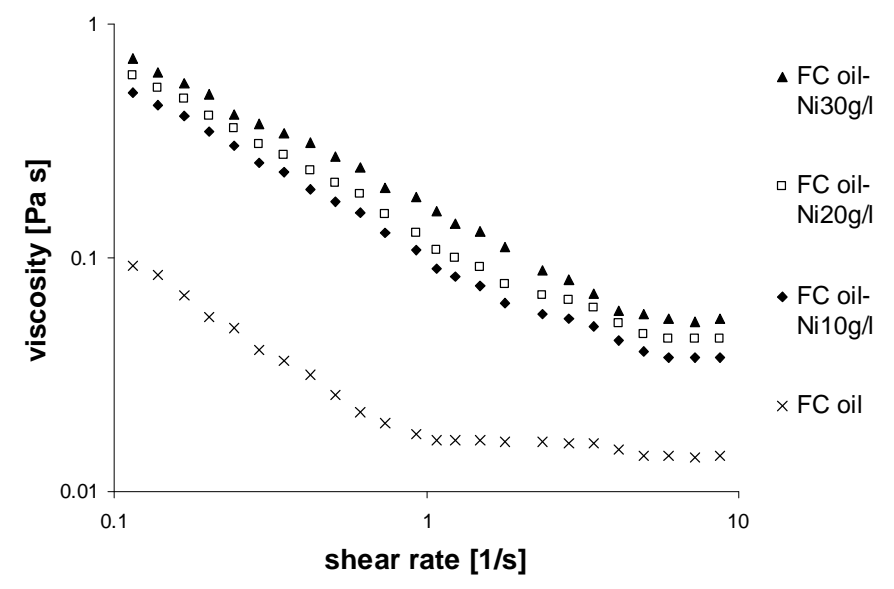

(c)

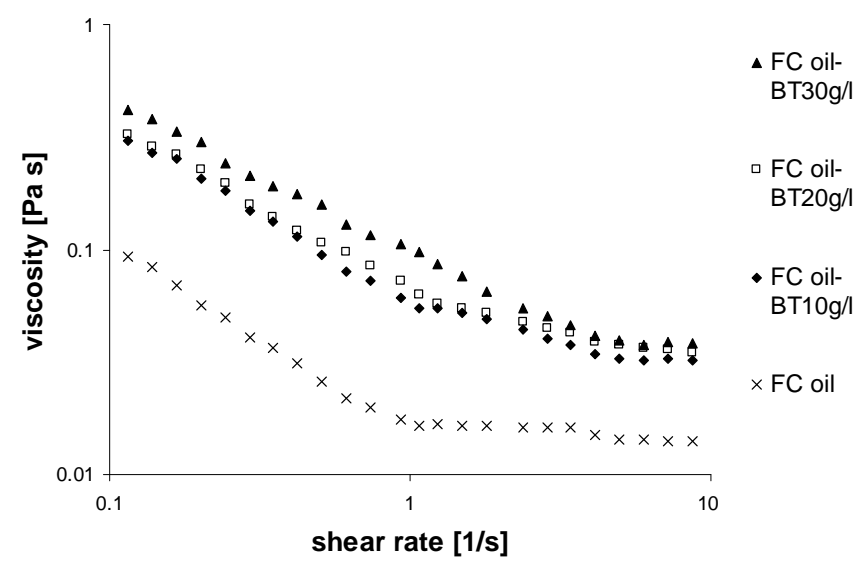

Figure 6 a-c 

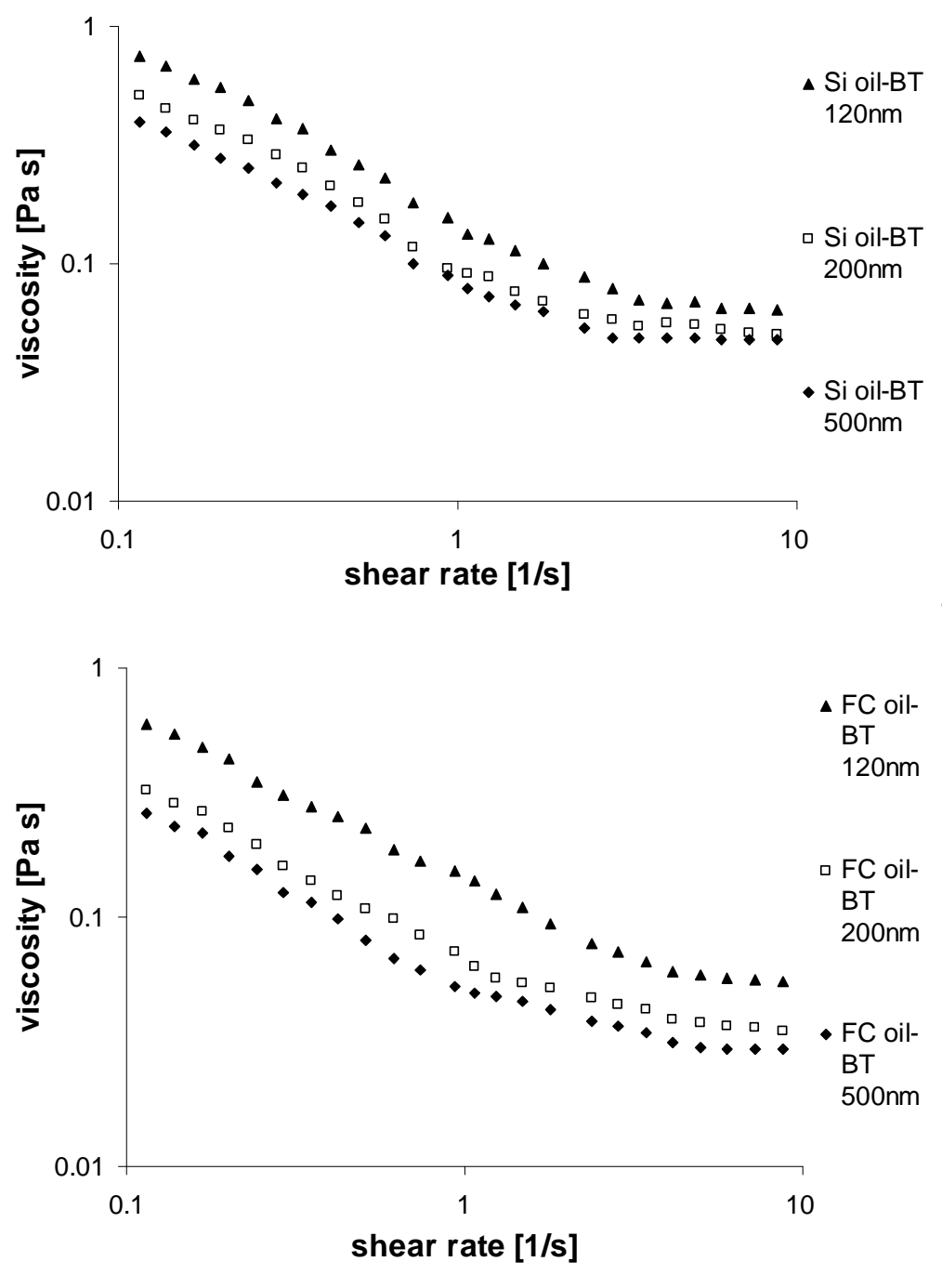

Figure $7 a-b$ 


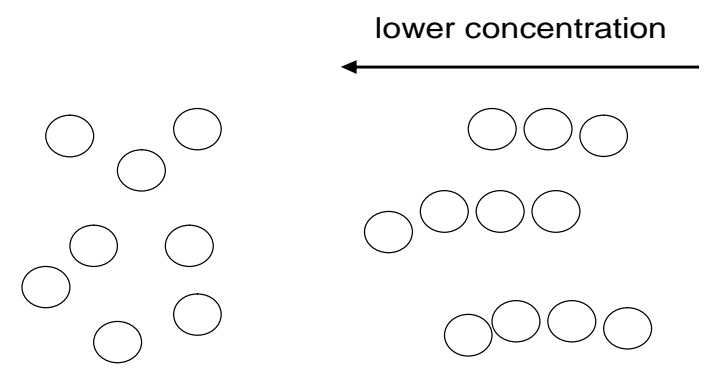

Dispersion of particles at low shear rate
Thinning due to formation of layers

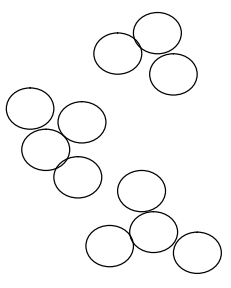

Formation of network

increase in applied shear

Figure 8 